\title{
Perioperative management of acute and chronic anemia: has the pendulum swung too far?
}

\author{
Gregory M. T. Hare, MD, PhD · James E. Baker, MD • \\ C. David Mazer, MD
}

Published online: 18 February 2009

(c) Canadian Anesthesiologists' Society 2009

\begin{abstract}
Anemia — and its treatment-contribute to morbidity and mortality. ${ }^{1}$ While chronic anemia is of multi-factorial etiology, acute anemia usually results from either acute hemorrhage (blood loss) or fluid resuscitation (hemodilution), or a combination of both. The increase in mortality associated with anemia has been best characterized in surgical patients with cardiovascular disease and in critically ill patients following neurotrauma. Cumulative evidence from clinical studies suggests that anemia-induced injury to vital organs, including the brain and kidney, may be responsible for the observed increase in mortality. The magnitude of this clinical problem is accentuated by evidence that one of the main treatment strategies for acute anemia, i.e., allogeneic blood transfusion, is also associated with an increase in mortality. ${ }^{2}$ Therefore, in order to improve patient outcomes, a clearer understanding is required of the mechanisms responsible for regulating organ perfusion and cell survival during anemia. ${ }^{1}$
\end{abstract}

\section{What's the problem with anemia?}

In simplistic terms, the key consequence of acute anemia is reduced blood $\mathrm{O}_{2}$ content leading to reduced tissue $\mathrm{O}_{2}$

G. M. T. Hare, MD, PhD (ه) · J. E. Baker, MD .

C. D. Mazer, MD

Department of Anesthesia, Cara Phelan Trauma Research Centre, Keenan Research Centre in the Li Ka Shing Knowledge Institute, St. Michael's Hospital, University of Toronto, 30 Bond

Street, Toronto, ON M5B 1W8, Canada

e-mail: hareg@smh.toronto.on.ca

G. M. T. Hare, MD, PhD . C. D. Mazer, MD

Department of Physiology, University of Toronto,

Toronto, ON, Canada delivery. Anemia can result in the development of tissue hypoxia. However, tissue hypoxia occurs in a hierarchical manner wherein organs that are critically important for survival, i.e., the heart and the brain, receive preferential oxygen delivery and maintain tissue "normoxia" at very low hemoglobin $(\mathrm{Hb})$ concentrations $\left.\left(\sim 35-40 \mathrm{~g} \cdot \mathrm{L}^{-1}\right)\right)^{3,4}$ Conversely, vital organs, such as the kidney and the intestine, become hypoxic at much higher $\mathrm{Hb}$ concentrations $\left.\left(\sim 60-70 \mathrm{~g} \cdot \mathrm{L}^{-1}\right)\right)^{3,4}$ Since arterial $\mathrm{PaO}_{2}$ and $\mathrm{Hb}$ saturation usually remain normal during anemia, different oxygen gradients are established between arterial blood and specific tissues. By contrast, hypoxemia is produced by reducing the fraction of inspired oxygen $\left(\mathrm{F}_{\mathrm{I}} \mathrm{O}_{2}\right)$ or by decreasing barometric pressure (high altitude). This reduces both the $\mathrm{PaO}_{2}$ and $\mathrm{Hb}$ saturation resulting in reduced blood-tissue oxygen gradients and global tissue hypoxia. While anemia and hypoxemia share the common element of producing tissue hypoxia, the two stimuli differ in the degree and distribution of tissue hypoxia. Thus, hypoxemia and anemia are different.

\section{Acute anemia: cardiovascular responses and distribution of perfusion}

Acute anemia provides a primary "hypoxic" stimulus by decreasing blood oxygen content and by suddenly reducing oxygen delivery to specific "hypoxic" organs, i.e., the kidney. ${ }^{3-5}$ Organ-specific tissue hypoxia or traditional chemoreceptor activation triggers a strong sympathetic response, thereby increasing heart rate, stroke volume, and cardiac output (CO). Concurrently, mean arterial blood pressure and systemic vascular resistance are reduced, while oxygen extraction is increased to optimize global tissue oxygen delivery. Interfering with either the increase 
in $\mathrm{CO}$ or the local cerebral vasodilatation disrupts cerebral tissue oxygen delivery leading to reduced brain tissue oxygen tension during acute hemodilution. ${ }^{1}$

The $\mathrm{CO}$ is differentially distributed in a hierarchical manner during anemia, such that critically vital organs, i.e., the brain and the heart, receive proportionally more oxygen relative to other vital organs, such as the kidney and the intestine. $^{3-5}$ This observation argues against the traditional rheological explanation which suggests a simple mathematical relationship between viscosity and blood flow whereby lower hematocrit (Hct) results in lower viscosity, reduced vascular resistance, and increased flow. Indeed, this reasoning formed the basis for acute hemodilution as a therapy in acute stroke, until subsequent studies demonstrated that the practice was ineffective. ${ }^{6}$ It is well known that there is a hierarchy of "protection" during shock states whereby the brain and heart are more "protected" than the kidney and the intestine. Although less appreciated, a similar "hierarchy" exists during acute anemia. ${ }^{3-5}$ The ability to improve the likelihood of survival during acute anemia will depend on our understanding of the mechanisms that optimize organ perfusion and protect cells from injury.

\section{Acute anemia: implications related to mortality and organ injury}

For decades clinicians have "topped up" anemic patients before surgery in the belief that anemia would worsen outcomes and, conversely, that transfusion would improve them. Indeed, anemia was identified over 20 years ago as an accurate predictor of mortality in all categories of surgical patients. $^{7}$ More recently, studies continue to demonstrate that anemia is a predictor of mortality in surgical patients, patients with cardiovascular disease, and patients with acute neurotrauma. ${ }^{1}$ The $\mathrm{Hb}$ threshold for increased mortality differs depending on patient condition and co-morbidities. The Transfusion Requirements in Critical Care (TRICC) trial demonstrated that critically ill patients can tolerate a $\mathrm{Hb}$ level near $70 \mathrm{~g} \cdot \mathrm{L}^{-1}$. However, specific patient populations, i.e., those suffering from neurotrauma and cardiovascular disease, experienced increased mortality and organ failure at much higher $\mathrm{Hb}$ thresholds. ${ }^{1,8,9}$ These patients may have better outcomes with higher $\mathrm{Hb}$ concentrations. The importance of maintaining optimal oxygen delivery is emphasized by recent clinical and experimental studies which demonstrate the increased likelihood of survival and improved clinical outcomes when blood oxygen content and tissue oxygen delivery are marginally increased at critical $\mathrm{Hb}$ thresholds. ${ }^{1,10}$

The synthesis of this data strongly supports the notion that we do not fully understand either the physiological mechanisms which regulate tissue oxygen delivery during anemia or the pathophysiological mechanisms which lead to increased organ injury and mortality. Furthermore, clinicians continue to be confounded by the fact that the most effective means of treating acute anemia, i.e., allogeneic blood transfusion, is also associated with increased mortality. This therapeutic dilemma emphasizes the importance of anemia prevention as a critical therapeutic strategy.

\section{Changes in transfusion thresholds}

Over the past several decades, clinicians have accepted progressively lower $\mathrm{Hb}$ levels in their patients because of the following beliefs: (1) anemia is well tolerated; (2) hemodilution optimizes blood rheology, favoring perfusion; (3) randomized trials show that lower $\mathrm{Hb}$ levels are safe; and (4) blood transfusion increases mortality. However, the $\mathrm{Hb}$ thresholds for organ injury and mortality are not well known. While some patients can tolerate a $\mathrm{Hb}$ level near $70 \mathrm{~g} \cdot \mathrm{L}^{-1}$, increased mortality and neurological injury may occur in others near this threshold. Indeed, the optimal $\mathrm{Hb}$ transfusion threshold may be even higher for patients suffering from neurotrauma or acute coronary syndromes. $^{11,12}$ This issue is urgent, as clinicians have witnessed notable paradigm shifts in the approaches to resuscitation of acutely ill anemic patients. However, this shift in patient management has been based on a rudimentary understanding of underlying mechanisms. A clearer understanding of the regulation of organ perfusion and cell survival during acute anemia is required in order to plan for successful advances in patient care.

\section{Pathophysiology of chronic anemia}

Chronic anemia is of multi-factorial etiology and has been associated with increased mortality ${ }^{13}$ and morbidity, including impairment of cognitive function ${ }^{14}$ and increased neurological injury. ${ }^{15}$ Also, treatment of acute and chronic anemia has been associated with improved cognitive abilities, supporting the concept that adequate brain tissue oxygen delivery is required to maintain optimal cerebral function. ${ }^{14,16,17}$ Indeed, correction of anemia has resulted in an improvement in the quality of life for a number of patient populations with chronic anemia. ${ }^{18-20}$

\section{Treatment strategies for acute and chronic anemia: pros and cons of erythropoietin therapy}

Clinicians today lack clear evidence to balance the risk of adverse outcomes of anemia against the possibility of 
favorable outcomes associated with its treatment. The risk of adverse outcomes and increased mortality associated with allogeneic blood transfusion would support the view that transfusion avoidance is preferable., ${ }^{21}$ In order to reduce the risk of both anemia and allogeneic transfusion, physicians have employed the use of erythropoietin (EPO). Initial EPO treatment strategies have produced promising results. EPO has been shown to increase $\mathrm{Hb}$ concentration, $^{22}$ to reduce allogeneic transfusion, ${ }^{22,23}$ to increase aerobic capacity and quality of life,${ }^{18-20}$ and to improve the likelihood of survival. ${ }^{24}$ In an attempt to further optimize these positive therapeutic outcomes, recent studies have targeted even higher Hct levels $(\sim 40 \%)$ with EPO therapy. ${ }^{19,25,26}$ Analysis of these studies demonstrated that this more aggressive approach did not always show improved benefit. Indeed, maintaining Hct levels near $40 \%$ may result in an increased incidence of adverse outcomes, including death, myocardial infarction, and stroke. ${ }^{19,26}$ These important negative outcomes occurred at Hct $\sim 40 \%$; therefore, they do not negate the previously reported advantages observed at lower target Hct values ( $\sim 30 \%$ ). As a result, many authors have concluded that we should not aim to normalize Hct $(\sim 40 \%)$; but rather, we should accept the benefit of partial correction of anemia with EPO therapy $(\mathrm{Hct} \sim 30 \%) .^{27,28}$ With this more conservative approach, the published benefits of EPO therapy may be gained while minimizing the risk of toxicity.

The established cytoprotective role of $\mathrm{EPO}^{1}$ may explain the observed increase in the likelihood of survival associated with EPO therapy in certain patient populations. ${ }^{24}$ However, this mechanism may also promote the progression of neoplastic disease. In addition, EPO has been shown to cause an increased incidence of thrombotic complications in the critically ill and in cancer patients. ${ }^{24,29}$ This prothrombotic effect was not observed in previous randomized trials when EPO was used acutely to correct anemia in surgical patient populations. ${ }^{30}$ However, in a recently completed, thus far unpublished trial in spine surgery (PROCRIT PR97-19-002; access ClinicalTrials.gov NCT 0021146), patients who were randomized to receive a high dose of EPO (up to $2400 \mathrm{U} \cdot \mathrm{kg}^{-1}$ in divided doses over 4 weeks) suffered an increased risk of deep vein thrombosis $(4.7 \%$ [16/340] vs $2.1 \%$ [7/340]). In this study, there was a significant risk associated with age (OR $2.63,>65 v s<65$ ) and gender (OR 0.42, females vs males). Despite an increase in thrombotic complications, the benefits of treatment included an increase in discharge $\mathrm{Hb}$ concentration (109 vs $100 \mathrm{~g} \cdot \mathrm{L}^{-1}$ ), a reduction in allogeneic blood transfusion (15.3 vs $25 \%, P=0.002$ ), and a total reduction (29.7 vs 55.0\%) of any type (autologous and allogeneic) of blood transfusion. Thus, high doses of EPO utilized in the perioperative period may be associated with an increased risk of thrombosis. However, the potential benefit of more moderate doses of EPO therapy must continue to be assessed in terms of improved quality of life, transfusion avoidance, and reduced mortality. These benefits may outweigh the risks of thrombosis in certain patient populations.

\section{Is anemia of chronic disease an adaptive response?}

Recently, the hypothesis that anemia of chronic disease may be an adaptive response has been proposed. ${ }^{31}$ While this hypothesis raises potentially interesting new approaches to the management of chronic anemia, it also raises some concerns: (1) The rationale for anemia of chronic disease being an adaptive response is not clearly developed in terms of its potential biological relevance; (2) Although causality between anemia and its associated morbidity and mortality has not been clearly established, the observed associations suggest that anemia is maladaptive or harmful; (3) Despite drug-related toxicity, partial treatment of acute and chronic anemia with EPO improves patient outcomes and quality of life; and (4) The assumption that severe acute anemia or hemodilution is well tolerated or even beneficial has reduced $\mathrm{Hb}$ thresholds for treatment and may have led to an increase in morbidity and mortality.

Sickle cell disease is an example of an adaptive response which favors survival and causes anemia. While the mutation in the $\mathrm{Hb}$ molecule may be protective from malaria, this genetic modification leads to anemia and associated maladaptive consequences, including an increase in the incidence of stroke. ${ }^{32}$ The risk of stroke can be reduced by treating anemia with blood transfusions. ${ }^{32}$ Thus, the concept of anemia of chronic disease being an adaptive process requires further development.

\section{Conclusions}

In conclusion, since both anemia and its treatment have been associated with increased mortality, further experimental and clinical studies are required to understand the cellular mechanisms involved. This information is needed to optimize treatment strategies in the perioperative and critical care settings for both acute and chronic anemia. It is premature to determine whether anemia of chronic disease is predominantly adaptive or maladaptive. Further evidence in support of the concept of "adaptive anemia" must be provided to ensure that the management of anemic patients is optimized. In addition, further randomized controlled trials employing rigorous methodological approaches are clearly warranted to assess the potential benefit of 
moderate dose EPO therapy in surgical patients with anticipated major blood loss.

\section{Prise en charge périopératoire de l'anémie aiguë et chronique: le retour du balancier est-il allé trop loin?}

L'anémie - et son traitement - jouent un rôle dans la morbidité et la mortalité. ${ }^{1}$ Alors que l'anémie chronique présente une étiologie multifactorielle, l'anémie aiguë est en général causée par une hémorragie aiguë (perte de sang) ou une réanimation liquidienne (hémodilution), ou une combinaison de ces deux éléments. L'augmentation de la mortalité associée à l'anémie a été décrite de la manière la plus précise chez les patients chirurgicaux souffrant de maladies cardiovasculaires et les patients sévèrement malades à la suite d'un traumatisme neurologique. Une quantité grandissante de données probantes tirées d'études cliniques suggère que les lésions provoquées par l'anémie aux organes vitaux, notamment au cerveau et aux reins, pourraient être à l'origine de l'augmentation de la mortalité observée. L'ampleur de ce problème clinique est encore accentuée par des données probantes soutenant que l'une des principales stratégies thérapeutiques pour traiter l'anémie aiguë, l'allotransfusion, est également associée à une mortalité accrue. ${ }^{2}$ Pour les raisons énumérées ci-dessus, une meilleure compréhension des mécanismes responsables de la régulation de la perfusion des organes et de la survie cellulaire pendant l'anémie est de mise si l'on souhaite améliorer les devenirs des patients. ${ }^{1}$

\section{Quel est le problème avec l'anémie?}

En quelques mots, la principale conséquence de l'anémie aiguë est un contenu d' $\mathrm{O}_{2}$ réduit dans le sang, ce qui provoque une libération réduite $\mathrm{d}^{\prime} \mathrm{O}_{2}$ dans les tissus. L'anémie peut provoquer l'hypoxie tissulaire. Toutefois, l'hypoxie tissulaire survient de façon hiérarchique : les organes les plus importants pour la survie, soit le cœur et le cerveau, bénéficient d'un apport préférentiel d'oxygène et maintiennent une « normoxie » tissulaire à des concentrations d'hémoglobine $(\mathrm{Hb})$ très basses $\left(\sim 35-40 \mathrm{~g} \cdot \mathrm{L}^{-1}\right){ }^{3,4}$ À l'inverse, les organes vitaux, comme les reins ou l'intestin, deviennent hypoxiques à des concentrations bien plus élevées d'hémoglobine $\left.\left(\sim 60-70 \mathrm{~g} \cdot \mathrm{L}^{-1}\right)\right)^{3,4}$ Étant donné que la $\mathrm{PaO}_{2}$ artérielle et la saturation de l'hémoglobine demeurent en général à un niveau normal pendant l'anémie, différents gradients d'oxygène s'établissent entre le sang artériel et les tissus spécifiques. Par contre, l'hypoxémie est provoquée en réduisant la fraction d'oxygène inspiré $\left(\mathrm{F}_{\mathrm{I}} \mathrm{O}_{2}\right)$ ou en abaissant la pression barométrique (haute altitude). Ces phénomènes réduisent à la fois la $\mathrm{PaO}_{2}$ et la saturation de l'hémoglobine, ce qui engendre des gradients d'oxygène sang-tissu plus bas et une hypoxie tissulaire globale. Bien que l'anémie et l'hypoxémie aient en commun la production d'hypoxie tissulaire, les deux stimuli se distinguent par le degré et la distribution de cette hypoxie tissulaire. Ainsi, l'hypoxémie et l'anémie sont deux choses différentes.

\section{L'anémie aiguë: réactions cardiovasculaires et distribution de la perfusion}

L'anémie aiguë fournit un stimulus « hypoxique » primaire en réduisant le contenu d'oxygène dans le sang et en réduisant soudainement l'apport d'oxygène à certains organes «hypoxiques » spécifiques, c'est-à-dire les reins. $^{3-5}$ L'hypoxie tissulaire spécifique à un organe et l'activation conventionnelle des chimiorécepteurs provoque une réaction sympathique soutenue, augmentant ainsi la fréquence cardiaque, le volume d'éjection et le débit cardiaque (DC). En même temps, la pression artérielle moyenne et la résistance vasculaire systémique sont réduites, tandis que l'extraction d'oxygène augmente pour maximiser l'apport global d'oxygène aux tissus. Toute interférence avec l'augmentation de DC ou la vasodilatation cérébrale locale interrompt l'apport d'oxygène au tissu cérébral, ce qui provoque une tension d'oxygène réduite dans le tissu cérébral pendant l'hémodilution aiguë. ${ }^{1}$

Le DC est distribué de façon différentielle et hiérarchique pendant l'anémie, de telle sorte que les organes vitaux les plus importants, soit le cerveau et le cœur, reçoivent proportionnellement plus d'oxygène comparativement aux autres organes vitaux comme les reins ou l'intestin. $^{3-5}$ Cette observation va à l'encontre de l'explication rhéologique conventionnelle selon laquelle il existe une relation mathématique simple entre la viscosité et le débit sanguin, où un hématocrite $(\mathrm{Hct})$ plus bas engendre une viscosité moindre, une résistance vasculaire réduite, et un débit accru. En fait, ce raisonnement a constitué le fondement du recours à l'hémodilution aiguë pour traiter les accidents vasculaires cérébraux (AVC) aigus jusqu'à ce que des études ultérieures montrent que cette pratique était inefficace. ${ }^{6}$ Le fait qu'il existe une hiérarchie de «protection » pendant les états de choc est bien connu : le cerveau et le cœur sont mieux «protégés » que les reins et l'intestin. Bien que moins connue, une « hiérarchie » semblable existe également pendant l'anémie aiguë. ${ }^{3-5} \mathrm{La}$ capacité d'améliorer la probabilité de survie pendant une anémie aiguë dépendra de notre compréhension des 
mécanismes qui maximisent la perfusion des organes et protègent les cellules des lésions.

\section{L'anémie aiguë: implications liées à la mortalité et à l'atteinte des organes}

Pendant des dizaines d'années, les cliniciens ont 'rempli' les patients anémiques avant qu'ils subissent une chirurgie, croyant que l'anémie provoquerait des devenirs pires et, à l'inverse, qu'une transfusion les améliorerait. Il est vrai que l'anémie a été identifiée il y a plus de vingt ans comme un indice précis de prédiction de la mortalité chez les patients subissant tous types de chirurgie. ${ }^{7}$ Plus récemment, des études ont démontré que l'anémie est un facteur prédictif de mortalité chez les patients subissant une chirurgie, les patients souffrant de maladies cardiovasculaires et les patients présentant un traumatisme neurologique aigu. ${ }^{1} \mathrm{Le}$ niveau d'hémoglobine correspondant à une mortalité accrue varie selon l'état et les co-morbidités des patients. L'étude TRICC (Transfusion Requirements in Critical Care) a démontré que les patients sévèrement malades pouvaient tolérer un niveau d'hémoglobine avoisinant $70 \mathrm{~g} \cdot \mathrm{L}^{-1}$. Néanmoins certaines populations spécifiques de patients, c'est-à-dire ceux souffrant de traumatisme neurologique ou de maladie cardiovasculaire, ont manifesté une mortalité accrue et une défaillance multisystémique à des seuils d'hémoglobine bien plus élevés. ${ }^{1,8,9}$ Ces patients pourraient présenter des devenirs améliorés à des concentrations plus élevées d'hémoglobine. L'importance du maintien d'un apport optimal d'oxygène est soulignée par plusieurs études cliniques et expérimentales récentes, lesquelles démontrent la probabilité accrue de survie et des devenirs cliniques améliorés lorsque le contenu d'oxygène dans le sang et l'apport d'oxygène aux tissus sont légèrement augmentés aux seuils d'hémoglobine cruciaux. ${ }^{1,10}$

La synthèse de ces données appuie la notion selon laquelle nous ne comprenons que partiellement les mécanismes physiologiques régulant l'apport d'oxygène aux tissus pendant l'anémie et les mécanismes physiopathologiques provoquant une mortalité et une atteinte multisystémique accrue. De plus, les cliniciens sont toujours déconcertés par le fait que la méthode la plus efficace pour traiter l'anémie aiguë, l'allotransfusion, est elle aussi associée à une mortalité accrue. Ce dilemme thérapeutique souligne l'importance de la prévention de l'anémie en tant que stratégie thérapeutique cruciale.

\section{Les changements de seuils de transfusion}

Au cours des dernières décennies, les cliniciens ont petit à petit accepté des niveaux d'hémoglobine plus bas chez leurs patients en raison des croyances suivantes : (1) l'anémie est bien tolérée ; (2) l'hémodilution optimise la rhéologie sanguine, favorisant ainsi la perfusion ; (3) des études randomisées montrent que des niveaux d'hémoglobine plus bas sont sécuritaires ; et (4) les transfusions sanguines augmentent la mortalité. Toutefois, les niveaux d'hémoglobine pour les atteintes multisystémiques et la mortalité ne sont pas bien connus. Alors que certains patients peuvent tolérer un niveau d'hémoglobine avoisinant $70 \mathrm{~g} \cdot \mathrm{L}^{-1}$ après la circulation extra-corporelle, chez d'autres la mortalité et les lésions neurologiques peuvent augmenter à ce seuil-là. En effet, le seuil optimal de transfusion d'hémoglobine peut atteindre des niveaux encore plus élevés chez les patients souffrant de traumatisme neurologique ou de syndrome coronarien aigu. ${ }^{11,12}$ Ce problème est urgent, les cliniciens ayant remarqué des transformations considérables des paradigmes selon l'approche de réanimation choisie pour les patients anémiques sévèrement malades. Toutefois, ce changement de la prise en charge des patients s'est fondé sur une compréhension rudimentaire des mécanismes sous-jacents. Une meilleure compréhension de la régulation de la perfusion des organes et de la survie cellulaire pendant l'anémie aiguë est nécessaire afin de préparer le terrain pour des progrès dans les soins prodigués aux patients.

\section{Physiopathologie de l'anémie chronique}

L'anémie chronique présente une étiologie multifactorielle et a été associée à une mortalité ${ }^{13}$ et une morbidité accrues, notamment une détérioration de la fonction cognitive ${ }^{14}$ et des atteintes neurologiques accrues. ${ }^{15}$ De plus, le traitement de l'anémie chronique et aiguë a été associé à des capacités cognitives améliorées, ce qui appuie l'hypothèse qu'un apport adéquat d'oxygène au cerveau est nécessaire pour maintenir une fonction cérébrale optimale. ${ }^{14,16,17}$ En effet, la correction de l'anémie a permis d'améliorer la qualité de vie de plusieurs populations de patients souffrant d'anémie chronique. ${ }^{18-20}$

\section{Stratégies thérapeutiques pour l'anémie aiguë et chronique: le pour et le contre du traitement à l'érythropoïétine}

À l'heure actuelle, les cliniciens n'ont pas de données probantes claires qui soupèsent le risque de devenirs indésirables de l'anémie contre la possibilité de devenirs favorables associés à son traitement. Le risque de devenirs indésirables et la mortalité accrue associés à l'allotransfusion appuieraient la perspective selon laquelle il est préférable d'éviter les transfusions. ${ }^{2,21}$ Aux fins de réduire 
le risque d'anémie et celui de l'allotransfusion, les médecins ont eu recours à l'érythropoïétine (EPO). Les premières stratégies thérapeutiques à l'EPO ont produit des résultats prometteurs. Il a été démontré que l'EPO augmente la concentration d'hémoglobine, ${ }^{22}$ réduit l'allotransfusion, ${ }^{22,23}$ augmente la capacité aérobique et la qualité de vie, ${ }^{18-20}$ et améliore la probabilité de survie. ${ }^{24}$ Tentant d'optimiser encore plus ces devenirs thérapeutiques positifs, certaines études récentes ont essayé d'atteindre des niveaux d'Hct encore plus élevés ( $40 \%$ ) avec un traitement à l'EPO. ${ }^{19,25,26}$ L'analyse de ces études a démontré que cette approche plus agressive ne donnait pas toujours une amélioration des bienfaits. En effet, le maintien des niveaux d'Hct autour de $40 \%$ a eu pour résultat une incidence accrue de devenirs défavorables, y compris le décès, l'infarctus du myocarde et l'AVC. ${ }^{19,26}$ Ces devenirs négatifs majeurs sont survenus lorsque l'Hct $\sim 40 \%$; pour cette raison, ils ne nient pas les avantages précédemment rapportés observés à des valeurs d'Hct cibles plus basses $(\sim 30 \%)$. Par conséquent, plusieurs auteurs ont conclu que nous ne devrions pas tenter de normaliser l'Hct ( $\sim 40 \%$ ); en revanche, nous devrions accepter les avantages d'une correction partielle de l'anémie avec le traitement à l'EPO (Hct 30\%). ${ }^{27,28}$ Cette approche plus conservatrice pourrait permettre de reproduire les bienfaits publiés du traitement à l'EPO tout en minimisant le risque de toxicité.

Le rôle cytoprotecteur de l'EPO est bien établi et pourrait expliquer l'augmentation observée de la probabilité de survie qui est associée avec le traitement de l'EPO chez certaines populations de patients. ${ }^{24}$ Cependant, ce mécanisme pourrait également favoriser la progression d'une maladie néoplasique. En outre, il a été démontré que l'EPO peut causer une incidence accrue de complications thrombotiques chez les patients sévèrement malades et chez les patients cancéreux. ${ }^{24,29}$ Cet effet prothrombotique n'a pas été observé dans les études randomisées précédentes, dans lesquelles l'EPO était utilisée précisément pour corriger l'anémie chez les patients subissant une chirurgie. ${ }^{30}$ Cependant, dans une étude réalisée récemment mais pas encore publiée en chirurgie du rachis (PROCRIT PR97-19002 ; pour y accéder ClinicalTrials.gov NCT 0021146), les patients randomisés à recevoir une dose élevée d'EPO (jusqu'à $2400 \mathrm{U} \cdot \mathrm{kg}^{-1}$ dans des doses divisées sur une période de 4 semaines) ont présenté un risque accru de thrombose veineuse profonde $(4,7 \%$ [16/340] vs $2,1 \%$ [7/340]). Dans cette étude, un risque significatif était associé à l'âge $(\mathrm{RC} 2,63,>65 v s<65)$ et au sexe $(\mathrm{RC} 0,42$, femmes $v s$ hommes). Malgré cette augmentation des complications thrombotiques, les bienfaits du traitement ont été une augmentation de la concentration d'hémoglobine au congé (109 vs $\left.100 \mathrm{~g} \cdot \mathrm{L}^{-1}\right)$, une réduction de l'allotransfusion $(15,3$ vs $25 \%, P=0,002)$, et une réduction totale
(29.7 vs $55.0 \%$ ) de tout type (autologue ou allogénique) de transfusion sanguine. Dès lors, l'utilisation de doses élevées d'EPO en période périopératoire pourrait être associée à un risque accru de thrombose. Néanmoins, le bienfait potentiel de doses plus modérées d'EPO doit continuer à être évalué en termes de qualité de vie améliorée, de prévention des transfusions et de mortalité réduite. Ces bienfaits pourraient l'emporter sur les risques de thrombose rencontrés chez certaines populations de patients.

\section{L'anémie des maladies chroniques est-elle une réponse adaptative?}

L'hypothèse que l'anémie des maladies chroniques pourrait constituer une réponse adaptative a récemment été émise. $^{31}$ Quoique cette hypothèse avance de nouvelles approches potentiellement intéressantes à la prise en charge de l'anémie chronique, certaines préoccupations y sont aussi liées : (1) La justification selon laquelle l'anémie des maladies chroniques est une réponse adaptative n'est pas clairement développée quant à sa pertinence biologique potentielle ; (2) Bien qu'un lien de cause à effet entre l'anémie et la mortalité et morbidité associées n'ait pas été clairement établi, les associations observées suggèrent que l'anémie est 'mal adaptative', voire nocive ; (3) Malgré la toxicité liée au médicament, un traitement partiel de l'anémie chronique et aiguë avec l'EPO améliore les devenirs des patients et leur qualité de vie ; et (4) L'hypothèse selon laquelle l'anémie aiguë grave ou l'hémodilution est bien tolérée, voire bénéfique, a mené à des seuils d'hémoglobine plus bas et provoqué une augmentation de la morbidité et de la mortalité.

L'anémie falciforme est un exemple de réponse adaptative qui favorise la survie et cause l'anémie. Quoique la mutation qui survient dans la molécule d'hémoglobine pourrait avoir un effet protecteur contre le paludisme, cette modification génétique provoque de l'anémie et des conséquences 'mal adaptatives' associées, y compris une augmentation de l'incidence d'AVC. ${ }^{32}$ Le risque d'AVC peut être réduit en traitant l'anémie à l'aide de transfusions sanguines. ${ }^{32}$ Dès lors, le concept selon lequel l'anémie des maladies chroniques est un processus adaptatif nécessite d'être approfondi.

\section{Conclusions}

En conclusion, étant donné que l'anémie et son traitement sont tous deux associés à une mortalité accrue, d'autres études expérimentales et cliniques sont nécessaires pour comprendre les mécanismes cellulaires impliqués. Si nous voulons optimiser les stratégies thérapeutiques dans les 
contextes périopératoire et des soins critiques pour l'anémie aiguë et chronique, ces informations sont nécessaires. À l'heure actuelle, il est encore trop tôt pour déterminer si l'anémie des maladies chroniques est plutôt adaptative ou 'mal adaptative'. Des données probantes supplémentaires appuyant le concept d'une « anémie adaptative » doivent être proposées pour garantir que la prise en charge des patients anémiques est optimisée. De plus, d'autres études randomisées contrôlées employant des approches méthodologiques rigoureuses sont clairement nécessaires pour évaluer le bienfait potentiel d'un traitement à base d'EPO à dose modérée chez les patients subissant une chirurgie chez qui l'on s'attend à des pertes sanguines majeures.

Acknowledgment The authors sincerely thank Drs. B.P. Kavanagh and S.S. Bolz for their critical review of this manuscript.

Conflicts of interest None declared.

\section{References}

1. Hare GM, Tsui AK, McLaren AT, Ragoonanan TE, Yu J, Mazer $C D$. Anemia and cerebral outcomes: many questions, fewer answers. Anesth Analg 2008; 107: 1356-70.

2. Hebert PC, Wells G, Blajchman MA, et al. A multicenter, randomized, controlled clinical trial of transfusion requirements in critical care. Transfusion Requirements in Critical Care Investigators, Canadian Critical Care Trials Group. N Engl J Med 1999; 340: 409-17.

3. van Bommel J, Trouwborst A, Schwarte L, Siegemund M, Ince C, Henny $C$. Intestinal and cerebral oxygenation during severe isovolemic hemodilution and subsequent hyperoxic ventilation in a pig model. Anesthesiology 2002; 97: 660-70.

4. van Bommel J, Siegemund M, Henny C, Ince C. Heart, kidney, and intestine have different tolerances for anemia. Transl Res 2008; 151: 110-7.

5. Johannes T, Mik EG, Ince C. Dual-wavelength phosphorimetry for determination of cortical and subcortical microvascular oxygenation in rat kidney. J Appl Physiol 2006; 100: 1301-10.

6. Asplund $K$. Haemodilution for acute ischaemic stroke. Cochrane Database Syst Rev 2006; (4): CD000103

7. Carson JL, Poses RM, Spence RK, Bonavita G. Severity of anaemia and operative mortality and morbidity. Lancet 1988; 1: 727-9.

8. Steyerberg EW, Mushkudiani N, Perel P, et al. Predicting outcome after traumatic brain injury: development and international validation of prognostic scores based on admission characteristics. PLoS Med 2008; 5: e165.

9. Young JB, Abraham WT, Albert NM, et al. Relation of low hemoglobin and anemia to morbidity and mortality in patients hospitalized with heart failure (insight from the OPTIMIZE-HF registry). Am J Cardiol 2008; 101: 223-30.

10. Murkin JM, Adams SJ, Novick RJ, et al. Monitoring brain oxygen saturation during coronary bypass surgery: a randomized, prospective study. Anesth Analg 2007; 104: 51-8.

11. Hebert PC, Yetisir E, Martin $C$, et al. Is a low transfusion threshold safe in critically ill patients with cardiovascular diseases? Crit Care Med 2001; 29: 227-34.

12. McIntyre LA, Fergusson DA, Hutchison JS, et al. Effect of a liberal versus restrictive transfusion strategy on mortality in patients with moderate to severe head injury. Neurocrit Care 2006; 5: 4-9.
13. Wu WC, Schifftner TL, Henderson WG, et al. Preoperative hematocrit levels and postoperative outcomes in older patients undergoing noncardiac surgery. JAMA 2007; 297: 2481-8.

14. Clarke SE, Jukes MC, Njagi JK, et al. Effect of intermittent preventive treatment of malaria on health and education in schoolchildren: a cluster-randomised, double-blind, placebocontrolled trial. Lancet 2008; 372: 127-38.

15. Karkouti K, Djaiani G, Borger MA, et al. Low hematocrit during cardiopulmonary bypass is associated with increased risk of perioperative stroke in cardiac surgery. Ann Thorac Surg 2005; 80: $1381-7$.

16. Pickett JL, Theberge DC, Brown WS, Schweitzer SU, Nissenson $A R$. Normalizing hematocrit in dialysis patients improves brain function. Am J Kidney Dis 1999; 33: 1122-30.

17. Weiskopf RB, Feiner J, Hopf HW, et al. Oxygen reverses deficits of cognitive function and memory and increased heart rate induced by acute severe isovolemic anemia. Anesthesiology 2002; 96: 871-7.

18. Littlewood TJ, Bajetta E, Nortier JW, Vercammen E, Rapoport B, Epoetin Alfa Study Group. Effects of epoetin alfa on hematologic parameters and quality of life in cancer patients receiving nonplatinum chemotherapy: results of a randomized, double-blind, placebo-controlled trial. J Clin Oncol 2001; 19: 2865-74.

19. Singh AK, Szczech $L$, Tang $K L$, et al. Correction of anemia with epoetin alfa in chronic kidney disease. N Engl J Med 2006; 355: 2085-98.

20. Soignet $S$. Management of cancer-related anemia: epoetin alfa and quality of life. Semin Hematol 2000; 37: 9-13.

21. Jani SM, Smith DE, Share D, et al. Blood transfusion and in-hospital outcomes in anemic patients with myocardial infarction undergoing percutaneous coronary intervention. Clin Cardiol 2007; 30: II49-56.

22. Feagan BG, Wong CJ, Kirkley A, et al. Erythropoietin with iron supplementation to prevent allogeneic blood transfusion in total hip joint arthroplasty. A randomized, controlled trial. Ann Intern Med 2000; 133: 845-54.

23. Corwin HL, Gettinger A, Pearl RG, et al. Efficacy of recombinant human erythropoietin in critically ill patients: a randomized controlled trial. JAMA 2002; 288: 2827-35.

24. Corwin HL, Gettinger A, Fabian TC, et al. Efficacy and safety of epoetin alfa in critically ill patients. N Engl J Med 2007; 357: 965-76.

25. Drueke TB, Locatelli F, Clyne N, et al. Normalization of hemoglobin level in patients with chronic kidney disease and anemia. N Engl J Med 2006; 355: 2071-84.

26. Besarab A, Bolton WK, Browne JK, et al. The effects of normal as compared with low hematocrit values in patients with cardiac disease who are receiving hemodialysis and epoetin. $\mathrm{N}$ Engl $\mathrm{J}$ Med 1998; 339: 584-90.

27. Remuzzi G, Ingelfinger JR. Correction of anemia-payoffs and problems. N Engl J Med 2006; 355: 2144-6.

28. Strippoli GF, Tognoni G, Navaneethan SD, Nicolucci A, Craig $J C$. Haemoglobin targets: we were wrong, time to move on. Lancet 2007; 369: 346-50.

29. Bennett CL, Silver SM, Djulbegovic B, et al. Venous thromboembolism and mortality associated with recombinant erythropoietin and darbepoetin administration for the treatment of cancer-associated anemia. JAMA 2008; 299: 914-24.

30. de Andrade JR, Frei D, Guilfoyle M. Integrated analysis of thrombotic/vascular event occurrence in epoetin alfa-treated patients undergoing major, elective orthopedic surgery. Orthopedics 1999; 22: s113-8.

31. Zarychanski R, Houston DS. Anemia of chronic disease: a harmful disorder or an adaptive, beneficial response? CMAJ 2008; 179: 333-7.

32. Lee MT, Piomelli S, Granger S, et al. Stroke prevention trial in sickle cell anemia (STOP): extended follow-up and final results. Blood 2006; 108: 847-52. 